\title{
Pediatric bladder neuroblastoma: Case report and literature review
}

\author{
Justin Zhu, BSc; Nathan A. Hoag; MD, Paul Gustafson, MD; Kourosh Afshar, MD; Andrew E. MacNeily, MD, FRCSC
}

Department of Urologic Sciences, University of British Columbia, Vancouver, BC

Cite as: Can Urol Assoc J 2013;7(9-10):e609-11. http://dx.doi.org/10.5489/cuaj.183 Published online September 10, 2013.

\section{Abstract}

Neuroblastoma (NBL) of the urinary bladder is an extremely rare diagnosis, with only 6 cases reported in the literature to date. We report the case of a 3-year-old boy who presented with gross hematuria, and was diagnosed with bladder NBL after partial cystectomy. Two-year follow-up has been unremarkable. This case highlights a rare neoplasm of the urinary bladder in a pediatric patient.

\section{Case report}

We report the case of a previously healthy 3-year-old boy who was referred to the pediatric urology service following a 1 -week history of gross hematuria. It was primarily described at the terminal portion of micturition, with no associated symptoms. Although physical examination was normal, ultrasound demonstrated a $1.6 \times 1.3 \times 2.2-\mathrm{cm}$ sessile, vascular mass on the anterosuperior bladder wall (Fig. 1).

Cystoscopy was performed demonstrating an "egg"-like tumour on the dome of the bladder of which biopsies were taken. Pathologic analysis was indeterminate, though suspicious for malignancy. The patient proceeded to have partial cystectomy, resecting the dome of the bladder with grossly negative margins and intra-operative frozen section concerning for rhabdomyosarcoma. The final pathologic diagnosis, however, was stroma-poor, differentiating neuroblastoma (NBL) with microscopic positive margins in a few foci (Fig. 2).

Mitotic karyorrhectic index (MKI) was low and histology was favourable on Shimada classification. Subsequent bone scan, bone marrow biopsy, mete-iodobenzoguanine (MIBG) labeled radionucleotide scan and urine catecholamines were all normal. No chemotherapy or radiation therapy was deemed necessary due to the low-risk nature of the tumour.
The patient was discharged in stable condition, and 2-year follow-up with ultrasound every 6 months has been unremarkable to date.

\section{Discussion}

NBL is the most common extra-cranial solid tumour in children and usually presents from birth to 6 years of age, with a median of 18 months. NBL arises embryologically from neural crest cells, and can arise anywhere along the sympathetic nervous system. ${ }^{1}$ Clinically, it usually presents in the adrenal glands (40\%), abdomen (15\%) and thorax $(15 \%) .^{2}$ The symptoms on presentation vary depending on site and extent of the disease, and range from organ-specific to non-specific constitutional symptoms, such as fever and malaise. Occasionally, patients can present asymptomatically for localized disease. Pelvic presentation occurs about $5 \%$ of the time. ${ }^{2}$

Only 6 previous cases of bladder NBL have been reported to date. ${ }^{3-7}$ Two other cases presented with gross hematuria. ${ }^{6,7}$ Of the remaining cases, 3 were asymptomatic, and detected as a result of a mass screening program, ${ }^{4,5}$ and one case was discovered as a palpable mass on physical examination. ${ }^{6}$ Five out of 6 reported cases of bladder NBL also occurred at the dome of the bladder, ${ }^{3-5,7}$ with one tumour arising from the anterior bladder wall. ${ }^{6}$

In four of the previously described cases, the patients underwent immediate partial cystectomy without neoadjuvant or adjuvant chemotherapy or radiation required. All were classified as stage 1, with favourable prognostic factors and preserved long-term survival noted. ${ }^{3-5}$

One 3-month old patient underwent partial cystectomy with negative surgical margins, but had poor-risk prognostic indicators, such as unfavourable Shimeda classification, n-myc amplification and $1 \mathrm{p}$ chromosome deletion, with lymph node extension. This patient was staged $2 \mathrm{~B}$, and subsequently observed. The patient had a lymph node recur- 


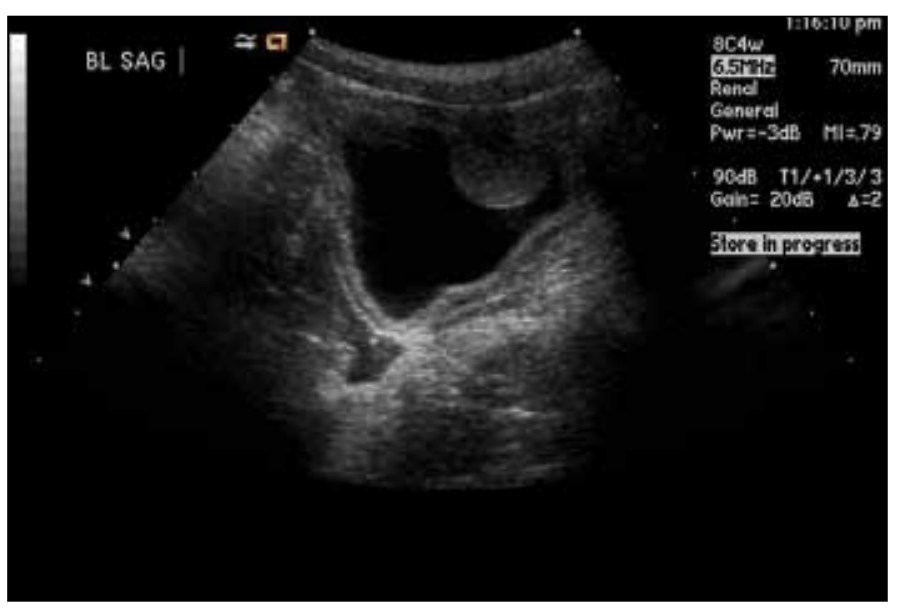

Fig. 1. Bladder ultrasound demonstrating $1.6 \times 1.3 \times 2.2-\mathrm{cm}$ sessile mass at bladder dome.

rence after 3 months, treated with surgical excision, followed by induction and then 6 -month maintenance chemotherapy and was disease free at the 30-month follow-up. ${ }^{6}$ In one case, vessel compression on imaging resulted in delayed surgery. The patient had neoadjuvant chemotherapy with 2 courses of carboplatin and etoposide, then 2 courses of cyclophosphamide, doxorubicin and vincristine. The patient then underwent partial cystectomy with negative margins and was well at 45 months follow-up. ${ }^{7}$ Other differential diagnoses for pediatric bladder masses include rhabdomyosarcoma, hemangioma, papilloma and carcinoma (urothelial and urachal). ${ }^{4}$

Patients with suspected NBL should have a complete history, physical examination and bloodwork, including complete blood count, serum chemistries, kidney function tests, liver function tests and serum or urinary catecholamine metabolite levels. The gold standard for diagnosis is histological confirmation based on biopsy of primary tumour or a large lymph node. Additional staging of disease may include a combination of diagnostic imaging tests, such as chest x-ray, abdominal ultrasound, computerized tomography $(\mathrm{CT})$, magnetic resonance imaging (MRI), MIBG scan and bone scan. MIBG scan is the current preferred imaging modality for assessing skeletal involvement of NBL. Bone marrow biopsies are indicated for assessment of bony metastasis. ${ }^{1}$ The patient in the presented case underwent ultrasound imaging and MIBG scan. Due to the favourable histologic characteristics of the tumour, axial imaging with CT or MRI was not undertaken.

NBL is classically staged from 1 to 4 , according to a revised international neuroblastoma staging system (INSS) developed in $1993 .^{8}$ In 2009, the International Neuroblastoma Risk Group (INRG) created a staging system that simplifies staging into L1, L2, M, and MS. ${ }^{9}$ Combining staging with other prognostic factors, such as age, Shimada grade, histology and genetics, allows patients to be grouped into 4

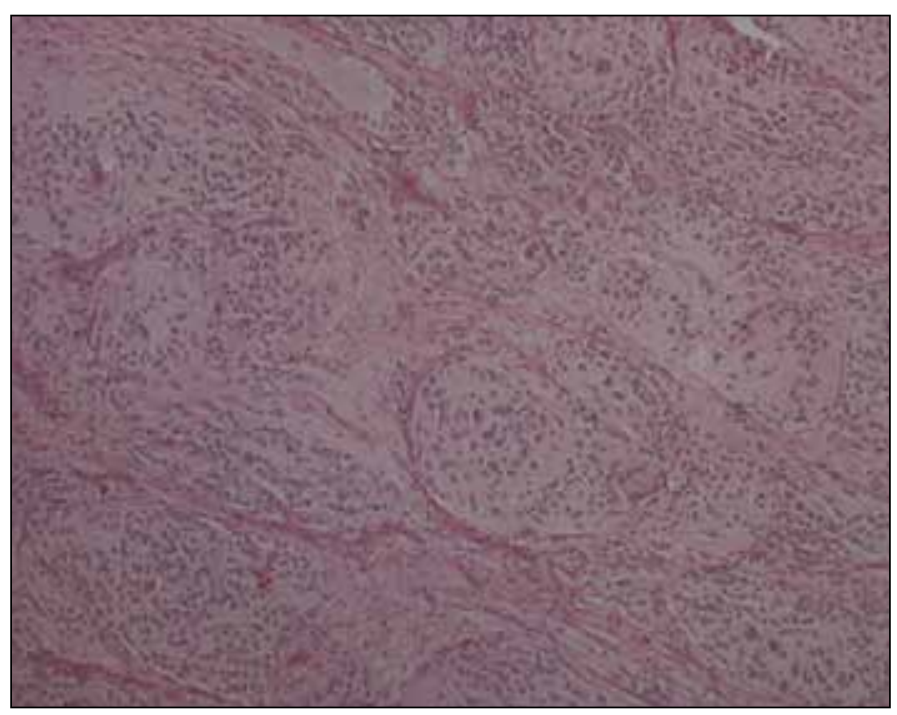

Fig. 2. A 10× magnification of stroma-poor, differentiating bladder neuroblastoma.

different prognostic risk groups ranging from very low, low, moderate and high. ${ }^{10,11}$

The patient presented in our case was stage 1 by INSS, and L1 by INRG based on the negative metastatic work-up. Combined with a favourable Shimada grade, non-amplification of MYCN and low MKI, our patient was in the very lowrisk group (group B) according to the INRG Staging System. Treatment of NBL depends on staging by INSS or INRG, and usually involves a combination of surgery, chemotherapy and/or radiation. Other treatments include hematopoietic stem cell transplantation and immunotherapy for treatment of more advanced disease. Typically, low-risk patients are treated with surgery alone or observation. Intermediate-risk patients are treated with neoadjuvant chemotherapy, with the amount and duration dictated by clinical and tumourspecific risk factors. Surgery is then undertaken. These treatment algorithms in the low- and intermediate-risk groups portend excellent survival outcomes greater than 95\%. Highrisk patients often undergo a combination of surgery, chemotherapy, radiation, hematopoietic stem cell transplant and immunotherapy with survival rates between $40 \%$ and $50 \%{ }^{1}$

\section{Conclusion}

We report the case of a 3-year-old boy presenting with hematuria who was found to have NBL in a rare location. With 7 cases of bladder NBL now reported to date, and perhaps other unreported cases, NBL should be considered as a possible differential diagnosis for bladder tumours in pediatric patients.

Competing interests: None declared. 
This paper has been peer-reviewed.

\section{References}

1. Owens C, Irwin M. Neuroblastoma: The impact of biology and cooperation leading to personalized treatments. Crit Rev Clin Lab Sci 2012;49:85-115. http://dx.doi.org/10.3109/10408363.2012.683483

2. Neuroblastic tumours of adrenal gland and sympathetic nervous system. Kleihues $P$, Cavenee $K$ (eds). Pathology \& Genetics of Tumours of the Nervous System. World Health Organization, IARC, Lyon, France; 2000:153.

3. Knoedler CJ, Kay R, Knoedler JP Sr, et al. Pelvic neuroblastoma. J Urol 1989;141:905-7.

4. lijir $R$, Tanaka $Y$, Kou $K$, et al. Bladder origin neuroblastoma detected by mass screening. Urol 1998;52:1139-41. http://dx.doi.org/10.1016/S0090-4295(98)00294-5

5. Yokoyama $S$, Hirakawa $H$, Ueno $S$, et al. Neuroblastoma of the urinary bladder, predinically detected by mass screening. Pediatrics 1999;103:e67. http://dx.doi.org/10.1542/peds.103.5.e67

6. Saez C, Marquez C, Quiroga E, et al. Neuroblastoma of the urinary bladder in an infant clinically detected by hematuria. Med Pediatr Oncol 2000;35:488-92. http://dx.doi.org/10.1002/1096911X(20001101)35:5<488::AID-MP08>3.0.C0;2-E
7. Entz-Werle N, Marcellin L, Becmeur F, et al. The urinary bladder: An extremely rare location of pediatric neuroblastoma. J Ped Surg 2003;38:e29. http://dx.doi.org/10.1016/S0022-3468(03)00291-4

8. Brodeur GM, Pritchard J, Berthold F, et al. Revisions of the international criteria for neuroblastoma diagnosis, staging, and response to treatment. J Clin Oncol 1993;11:1466.

9. Monclair T, Brodeur GM, Ambros PF, et al. The International Neuroblastoma Risk Group (INRG) Staging System: An INRG Task Force Report. J Clin Oncol 2009;27:298-303. http://dx.doi.org/10.1200/ JC0.2008.16.6876

10. Shimada H, Ambros IM, Dehner LP, et al. The International Neuroblastoma Pathlogy Classification (the Shimada System). Cancer 1999;86:364-72. http://dx.doi.org/10.1002/(SICI) 10970142(19990715)86:2<364::AID-CNCR21>3.0.C0;2-7

11. Cohn SL, Pearson ADJ, London WB, et al. The International Neuroblastoma Risk Group (INRG) Classification System: An INRG Task Force Report. J Clin Oncol 2009;27:289-97. http://dx.doi.org/10.1200/ JC0.2008.16.6785

Correspondence: Dr. Nathan Hoag, UBC Department of Urologic Sciences, Level 6 - 2775 Laurel St., Vancouver, BC V5Z 1M9; nathanhoag2@gmail.com 